\title{
DENSITIES ON LOCALLY COMPACT ABELIAN GROUPS
}

\author{
BY I. D. BERG ${ }^{1}$ AND L. A. RUBEL ${ }^{2}$
}

Communicated by Paul Cohen, November 1, 1967

A density on a locally compact Abelian group $G$ is a bounded system of compatible measures on the compact quotients of $G$. The notion of density arises naturally both in connection with the theory of uniform distribution and with the theory of almost periodic functions. Our main result effectively answers the question "when are all densities on $G$ induced by measures on some compactification of $G$ ?" This turns out to be equivalent to the question "when does each density on $G$ extend to a bounded linear functional on the space of semiperiodic functions on $G$ ?"

Let $G$ be a locally compact Abelian (LCA) group.

Definition. A density $\mu$ on the LCA group $G$ is a system of measures on the compact quotients of $G$ constructed as follows. To each compact quotient $Q$ of $G$ is associated a regular Borel measure $\mu_{Q}$ defined on the Borel sets of $Q$ and satisfying

(1) If $E$ is a Borel set of $Q$ and if $Q$ is a quotient of $Q^{\prime}$ then $\mu_{Q}(E)$ $=\mu_{Q^{\prime}}\left(\phi^{-1}(E)\right)$, where $\phi: Q^{\prime} \rightarrow Q$ is the natural homomorphism.

$$
\|\mu\|=\operatorname{def} \sup _{Q}\left\{\left\|\mu_{Q}\right\|\right\}<\infty .
$$

An important example of a density is Haar density $\lambda$, where $\lambda_{Q}$ is simply Haar measure on $Q$. The densities form a commutative Banach algebra under the obvious operations of addition, scalar multiplication, and convolution $*$ defined by $(\mu * \nu)_{Q}=\mu_{Q} * \nu_{Q}$.

If $G$ is an LCA group and $H$ is a closed subgroup of $G$ such that $G / H$ is compact, then we say that $H$ is a subgroup of compact index in $G$. We say that a function $f$ on $G$ is periodic if $f$ is constant on the cosets of some subgroup $H$ of compact index, and we then call $H$ a period of $f$. It is easy to see that a continuous, complex-valued, periodic function on $G$ is necessarily an almost periodic function, and it can be shown easily that an almost periodic function is periodic if and only if the characters which occur in its Fourier expansion generate a discrete subgroup of the dual group $G^{\wedge}$ of $G$. In particular, a continuous character $\chi$ is periodic if and only if the group generated by $\chi$ is a discrete subgroup of $G^{\wedge}$.

${ }^{1}$ The research of the first author was partially supported by National Science Foundation grant number NSF GP 5370.

${ }^{2}$ The research of the second author was partially supported by United States Air Force Office of Scientific Research grant number AFOSR 460-67. 
We denote by $P(G)$ the space of continuous complex-valued periodic functions on $G$. In general, $P(G)$ is not a vector space. If $f \in P(G)$ and if $\mu$ is a density then we define $\int f d \mu$ by $\int f d \mu=\int_{Q} f_{Q} d \mu_{Q}$ where $f$ has period $H$ with $Q=G / H$ and $f_{Q}$ is defined as a function on $Q$ by $f_{Q}(x+H)=f(x)$. That $\int f d \mu$ is well defined follows from condition $(1)$ in the definition of a density. Hence, the densities may be regarded as the bounded complex-valued functionals on $P(G)$ which are linear on the subspace of functions of any one fixed period.

We denote by $C_{p}(G)$ the vector space of finite linear combinations of complex-valued periodic functions, and we call the uniform closure of $C_{p}(G)$ the space of semiperiodic functions on $G$.

LEMma 1. If $f_{1}, f_{2}, \cdots, f_{n}$ are elements of $P(G)$ then $\mu\left(\sum_{i=1}^{n} f_{i}\right)$ $=\sum_{i=1}^{n} \mu\left(f_{i}\right)$ extends each density $\mu$ to a well-defined but possibly unbounded linear functional on $C_{p}(G)$, with

$$
\left|\mu\left(\sum_{i=1}^{n} f_{i}\right)\right| \leqq 2^{n+1}\|\mu\|\left\|\sum_{i=1}^{n} f_{i}\right\|_{\infty} .
$$

Our main result characterizes those LCA groups $G$ for which each density is actually bounded on $C_{p}(G)$ and hence extends to an element of the conjugate space of the space of semiperiodic functions.

TheOREM 1. Each density on $G$ extends to a bounded linear functional on $C_{p}(G)$ if and only if the sum of any two periodic continuous characters of $G$ is periodic.

By $\bar{G}^{p}$ we denote the compact dual of the discrete group generated by the continuous periodic characters of $G$. It is easy to see that every bounded Borel measure on $\bar{G}^{p}$ induces a density on $G$. We call $\bar{G}^{p}$ the semiperiodic compactification of $G$.

THEOREM 2. Each density on $G$ is induced by a measure on $\bar{G}^{p}$ if and only if the sum of any two periodic characters of $G$ is periodic. In this case, the algebra $D(G)$ of all densities is Banach-algebra isomorphic and isometric to the algebra $M\left(\bar{G}^{p}\right)$ of all bounded Borel measures on $\bar{G}^{p}$.

THEOREM 3. The sum of any two periodic continuous characters of $G$ is periodic if and only if either $G$ is totally disconnected, or $G$ has no $\boldsymbol{R}^{n}$ part and each discrete quotient of $G$ is of bounded order.

There exist LCA groups on which the sum of any two periodic continuous characters is periodic but for which there exist two periodic continuous functions whose sum is not periodic. The condition that the sum of any two periodic characters be periodic can be expressed 
in terms of the structure of the system of compact quotients of $G$. We say that two subgroups $H_{1}, H_{2}$ of compact index in $G$ are incident if $H_{1} \cup H_{2}$ is contained in a proper subgroup of compact index. Two subgroups $H_{1}, H_{2}$ of compact index are independent if there is no third subgroup $H$ of compact index that is incident both to $H_{1}$ and $\mathrm{H}_{2}$. It is not hard to show that the sum of any two periodic continuous characters of $G$ is periodic if and only if there exist no two independent subgroups of $G$ of compact index.

If $D(G)=M\left(\bar{G}^{p}\right)$ then it is clear that each real density on $G$ has a decomposition as the difference of two positive densities. But, for example, if $G=R$, then each real density decomposes, and yet we have $D(G) \neq M\left(\bar{G}^{p}\right)$. This shows that there are positive densities on $\boldsymbol{R}$ that are not induced by measures on $\bar{R}$, the Bohr compactification of $R$, which coincides in this case with the semiperiodic compactification.

TheOREM 4. There is a real density on the cylinder group $R \times T$ which is not the difference of two positive densities.

Our proofs depend rather heavily on the structure theorem for LCA groups, and some of them are long and complicated. There is not space in this announcement to describe them, and we shall present them elsewhere.

UNIVERSTTY OF ILLINOIS AND

INSTITUTE FOR AdVANCED STUDy 